Research Paper

\title{
Rhein Protects against Obesity and Related Metabolic Disorders through Liver X Receptor-Mediated Uncoupling Protein 1 Upregulation in Brown Adipose Tissue
}

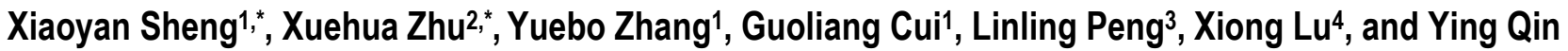 \\ Zang $^{1, \otimes}$
}

1. Key Laboratory of Nutrition and Metabolism, Institute for Nutritional Sciences, Shanghai Institutes for Biological Sciences, Graduate School of CAS, Chinese Academy of Sciences, Shanghai 200031, China

2. Shanghai Key Laboratory of Signaling and Disease Research, School of Life Science and Technology, Tongji University, 1239 Siping Road, Shanghai 200092, China

3. State Key Laboratory of Medical Genomics and Shanghai Institute of Hematology, Rui Jin Hospital Affiliated to Shanghai Jiao Tong University School of Medicine, Shanghai 200025, China

4. Laboratory of Cell Biology and Histopathology, Shanghai University of Traditional Chinese Medicine, Shanghai 201203, China

* These authors contributed equally to this work.

Corresponding author: Dr. Ying Qin Zang, Key Laboratory of Nutrition and Metabolism, Institute for Nutritional Sciences, Shanghai Institutes for Biological Sciences, Graduate School of CAS, Chinese Academy of Sciences, Shanghai 200031, China. Tel: +86-21-61590908 Fax: +86-21-61590700 E-mail: yqin@sibs.ac.cn.

(C) Ivyspring International Publisher. This is an open-access article distributed under the terms of the Creative Commons License (http://creativecommons.org/ licenses/by-nc-nd/3.0/). Reproduction is permitted for personal, noncommercial use, provided that the article is in whole, unmodified, and properly cited.

Received: 2012.05.10; Accepted: 2012.10.18; Published: 2012.10.29

\begin{abstract}
Liver X receptors (LXRs) play important roles in regulating cholesterol homeostasis, and lipid and energy metabolism. Therefore, LXR ligands could be used for the management of metabolic disorders. We evaluated rhein, a natural compound from Rheum palmatum L., as an antagonist for LXRs and investigated its anti-obesity mechanism in high-fat diet-fed mice. Surface plasmon resonance assays were performed to examine the direct binding of rhein to LXRs. LXR target gene expression was assessed in 3T3-LI adipocytes and HepG2 hepatic cells in vitro. C57BL/6J mice fed a high-fat diet were orally administered with rhein for 4 weeks, and then the expression levels of LXR-related genes were analyzed. Rhein bound directly to LXRs. The expression levels of LXR target genes were suppressed by rhein in 3T3-LI and HepG2 cells. In white adipose tissue, muscle and liver, rhein reprogrammed the expression of LXR target genes related to adipogenesis and cholesterol metabolism. Rhein activated uncoupling protein I (UCPI) expression in brown adipose tissue (BAT) in wild-type mice, but did not affect UCPI expression in LXR knockout mice. In HIB-IB brown adipocytes, rhein activated the UCPI gene by antagonizing the repressive effect of LXR on UCPI expression. This study suggests that rhein may protect against obesity and related metabolic disorders through LXR antagonism and regulation of UCPI expression in BAT.
\end{abstract}

Key words: LXR; diet-induced obesity; UCP1; antagonist; rhein.

\section{Introduction}

Our modern lifestyle, which is characterized by an overabundant supply of food rich in energy and little physical activity, is increasing the prevalence of obesity (1). Obesity is considered to be the central and causal component of multiple metabolic disorders, including dyslipidemia, hypertension, heart disease 
and type 2 diabetes (2-5). Obesity is associated with excessive accumulation of fat. In mammals, fat is stored in two different types of adipose tissues: white adipose tissue (WAT) and brown adipose tissue (BAT). WAT is composed of large lipid droplets and acts as the main energy store (6). By contrast, BAT contains small lipid droplets and is the main site of fat metabolism that serves to increase energy expenditure in the form of thermogenesis. This process is mediated by the unique and high expression of uncoupling protein 1 (UCP1), a protein that uncouples energy from ATP production to produce heat $(7 ; 8)$. Following the first report of BAT in adult humans by Nedergaard et al., three independent research groups have since shown that adults have active BAT in their neck and upper chest regions that could be activated by cold exposure (9-12). Interestingly, Virtanen et al. estimated that activating $63 \mathrm{~g}$ of supraclavicular BAT could burn an amount of energy equivalent to approximately $4.1 \mathrm{~kg}$ of adipose tissue over 1 year in one of their subjects (10). Therefore, targeting BAT in adults may offer an effective treatment for obesity and related metabolic disorders.

Liver $X$ receptors (LXRs) are nuclear receptors that heterodimerize with retinoid $X$ receptors (RXRs) and bind to LXR-responsive elements (LXREs) containing direct repeats (DRs) of the core sequence AGGTCA separated by four nucleotides (DR-4). Two genes ( $\alpha$ and $\beta$ ) encode two LXR proteins. LXRa is highly expressed in the liver while LXR $\beta$ is ubiquitously expressed (13-15). Once bound to its ligand, LXR undergoes a conformational change, recruits coactivator proteins and enables transcription of its target genes (16). LXRs are well known for their ability to regulate cholesterol, fatty acid and glucose metabolism (17-20). A recent study showed that LXRa is a direct transcriptional inhibitor of UCP1 and thus negatively regulates the expression of UCP1 and the manifestation of the brown adipocyte phenotype to ultimately reduce energy expenditure (21). Taken together, LXRs seem to offer a potential pharmacological target to treat or inhibit the development of metabolic syndrome because of their multiple roles in cholesterol, lipid, carbohydrate and energy metabolism.

Rhein (4,5-dihydroxyanthraquinone-2-carboxylic acid) is an anthraquinone and is one of the major components of Rheum palmatum L., which has been used for weight control in Chinese medicine. Some studies have shown that rhein enhances insulin sensitivity and glucose regulation in animals (22-24), and inhibits cell proliferation and apoptosis in many cell lines (25-30). Furthermore, diacerhein and rhein (a metabolite of diacerhein that retains its biological ac- tivity) reduced the severity of osteoarthritis in men, and diacerhein is now an established treatment for osteoarthritis (31-34). Our recent study showed that rhein has beneficial effects on nonalcoholic fatty liver disease (NAFLD) in high-fat diet-induced obesity by promoting a negative energy balance, improving hepatic lipogenic regulation, and exerting immunomodulatory effects (35). However, its anti-obesity mechanism in high-fat diet-fed mice remains largely unknown and needs to be further investigated. Therefore, in this study, we aim to study the underlying mechanisms involved in the role of rhein in ameliorating NAFLD.

\section{Results}

\section{Rhein binds directly to $L X R \alpha$ and $L X R \beta$}

In our previous study, we reported that rhein ameliorated NAFLD and associated disorders through an LXR-mediated negative energy balance (35). To identify whether rhein binds directly to LXRs, we performed an in vitro screening assay based on the surface plasmon resonance (SPR) method. This assay was confirmed to be specific, dose dependent and reproducible for the analysis of interactions between small molecular compounds and proteins (36-38). Using this assay, we found that rhein dose-dependently bound to LXRa and LXR $\beta$ with $\mathrm{K}_{\mathrm{D}}$ values of $46.7 \mu \mathrm{M}$ and $31.97 \mu \mathrm{M}$, respectively (Figure $1 \mathrm{C}$ and $1 \mathrm{~F}$ ). These low binding affinities closely correlate with the characteristics of the nuclear receptors (16). To confirm the binding specificity of rhein, we examined the abilities of GW3965 (as a positive control) and a herbal compound puerarin (as a negative control) to bind to the LXRs. GW3965 at $1 \mu \mathrm{M}$ bound significantly to the LXRs (Figure 1A and $1 D$ ), whereas no binding was detected with puerarin, even when the dose was increased to $100 \mu \mathrm{M}$ (Figure $1 \mathrm{~B}$ and $1 \mathrm{E}$ ). In addition, to investigate whether rhein regulates the transcriptional activity of other nuclear receptors related to lipid, cholesterol and glucose metabolism, we performed an in vitro screening assay based on luciferase activity. By fusing GAL4 to the ligand-binding domain (LBD) of the nuclear receptors, we found that $12.5 \mu \mathrm{M}$ rhein significantly inhibited the transcriptional activities of LXRa and LXR $\beta$ but did not affect the transcriptional activities of other nuclear receptors (Supplementary Material: Figure S1). These data indicate that rhein acts as an antagonist of LXRs by directly binding to both LXRa and LXR $\beta$.

\section{Rhein decreases the expression of LXR target genes in vitro}

To verify whether rhein decreases the expres- 
sion of LXR target genes via LXR antagonism, we used two cell models, 3T3-L1 adipocytes differentiated for 7 days and high level LXR-expressing HepG2 cells. After incubation with dimethyl sulfoxide (DMSO) alone, $1 \mu \mathrm{M}$ GW3965 alone or $1 \mu \mathrm{M}$ GW3965 and $25 \mu \mathrm{M}$ rhein for $48 \mathrm{~h}$, the cells were collected for real-time reverse-transcriptase polymerase chain reaction (RT-PCR) analysis. In differentiated 3T3-L1 adipocytes, $1 \mu \mathrm{M}$ GW3965 markedly increased the expression of LXR target genes related to cholesterol metabolism [ATP-binding cassette, sub-family A (ABC1), member 1 (ABCA1) and ATP-binding cassette, sub-family G (WHITE), member 1 (ABCG1)] and adipogenesis [sterol regulatory element binding transcription factor 1 (SREBP1c), fatty acid synthase (FAS), stearoyl-Coenzyme A desaturase 1 (SCD1) and acetyl-CoA carboxylase 1 (ACC1)], but did not affect the expression of a gene related to glucose metabolism [glucose transporter 4 (GLUT4)]. Rhein $(25 \mu \mathrm{M})$ significantly inhibited the increased expression of LXR target genes induced by GW3965 (Figure 2A). Similar effects were observed in HepG2 cells (Figure 2B). These data indicate that although rhein has a lower binding affinity compared with GW3965 (Figure 1), once bound, rhein induces a strong effect.

To identify whether rhein can affect the differentiation or apoptosis of adipocytes, 3T3-L1 cells were induced to differentiate by insulin, dexamethasone and 3-isobutyl-1-methylxanthine (IBMX), and were then treated with DMSO or rhein. We found that rhein did not significantly affect 3T3-L1 cell differentiation (Supplementary Material: Figure S2A and S2B) or apoptosis (Supplementary Material: Figure S2B).
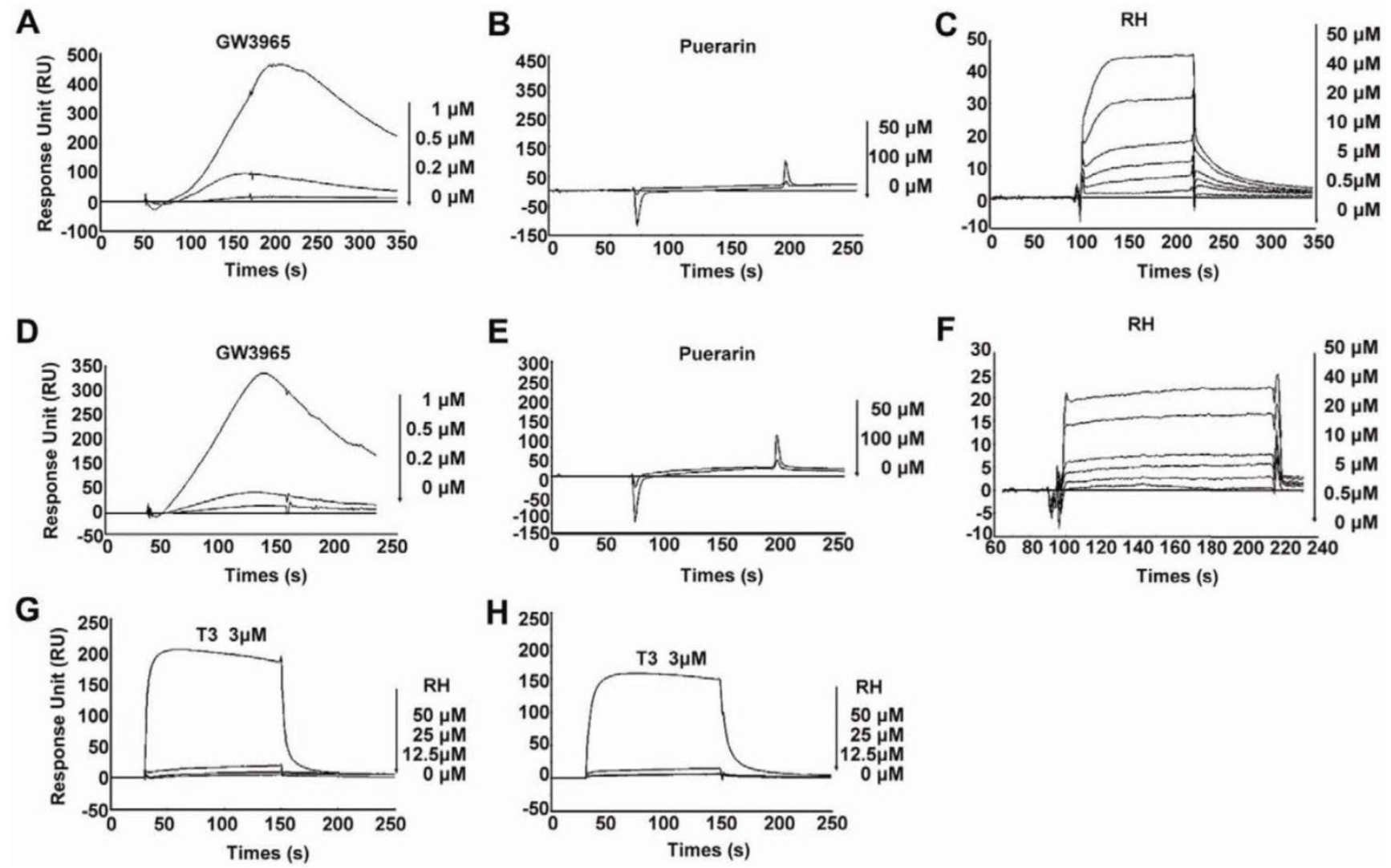

Figure I Rhein binds directly to liver X receptors (LXRs). (A) to (F) Specific binding affinities to the ligand-binding domains (LBDs) of LXRs were analyzed by surface plasmon resonance (SPR) assays. The photographs were obtained after injection of a series of concentrations of GW3965, puerarin or rhein in phosphate-buffered saline (PBS) containing $0.2 \%$ dimethyl sulfoxide (DMSO) over the immobilized LXRa-LBD surface (A to $\mathbf{C}$ ) or the LXR $\beta$-LBD surface (D to F) on a CM5 sensor chip. The $K_{D}$ values of rhein for $L X R \alpha$ and $L X R \beta$ were $46.7 \mu M$ and $3 I .97$ $\mu M$, respectively. $(\mathbf{G})$ and $(\mathbf{H})$ Specific binding affinities against thyroid hormone receptors (TRs) were analyzed by SPR assays. The photographs were obtained after injection of a series of concentrations of rhein and T3 in PBS containing $0.2 \%$ DMSO over the immobilized TRa surface (G) or TR $\beta$ surface $\mathbf{( H )}$ on a CM5 sensor chip. For the SPR assay, ligand binding was measured at a flow rate of $30 \mu \mathrm{L} / \mathrm{min}$ for $2 \mathrm{~min}$, and dissociation was initiated upon replacement of the analyte with running buffer. The response units (RU) were corrected for non-specific binding to a blank flow channel (relative response). $\mathrm{RH}$, rhein. 
A
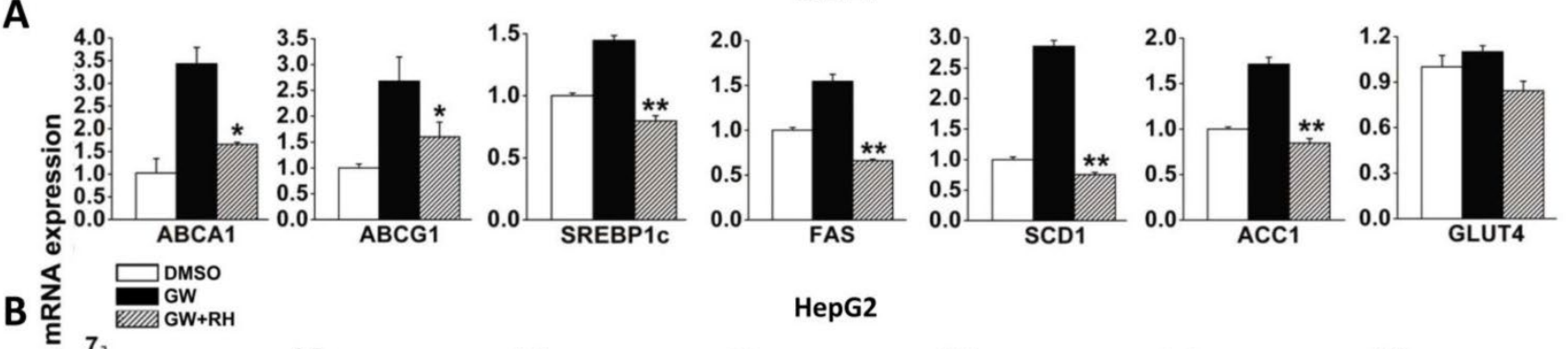

HepG2
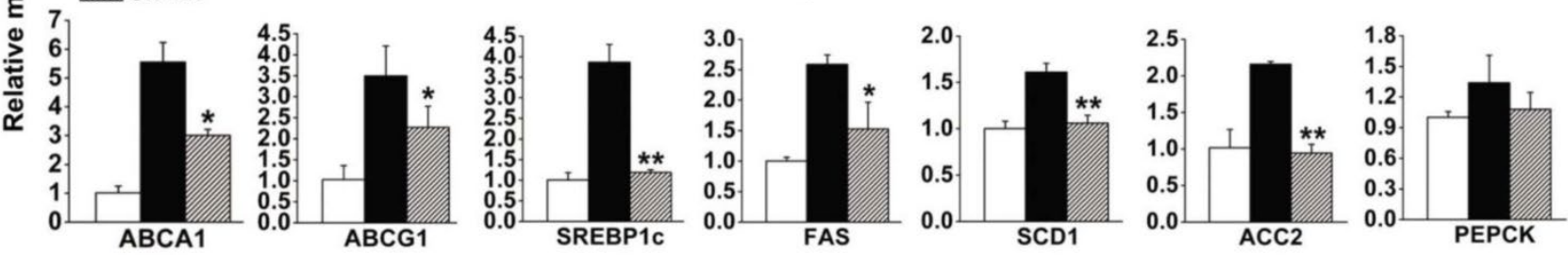

Figure 2 Rhein decreases liver $X$ receptor (LXR) target gene expression in vitro. Rhein decreased the expression of $L X R$ target genes in 3T3-LI adipocytes (A) and HepG2 cells (B). White bars, 3T3-LI adipocytes or HepG2 cells treated with dimethyl sulfoxide (DMSO); black bars, 3T3-LI adipocytes or HepG2 cells were treated with I $\mu M$ GW3965 (GW); striped bars, 3T3-LI adipocytes or HepG2 cells were treated with I $\mu \mathrm{M}$ GW3965 and $25 \mu \mathrm{M}$ rhein $(\mathrm{RH})$. mRNA expression levels were estimated by quantitative real-time PCR. The results are shown as mRNA levels relative to that treated with DMSO. Values are means \pm standard deviation of three independent experiments.

\section{Rhein reprograms the expression of LXR tar- get genes in WAT, muscle and liver}

To further explore the molecular mechanisms by which rhein protects against obesity and improves lipid and glucose metabolism (35), we performed quantitative real-time PCR to analyze the expression levels of genes in WAT, muscle and liver, which are highly metabolic tissues. Mice fed a high-fat diet and treated with or without rhein were used in this analysis. In WAT, we found that the expression levels of LXR target genes related to adipogenesis (SREBP1c and FAS) were significantly decreased, while the expression levels of SCD1 and ACC1 were non-significantly decreased. Rhein did not significantly affect the expression of GLUT4 or insulin receptor substrate 1 (IRS1). Rhein also decreased the expression of LXR target genes related to cholesterol metabolism (ABCA1 and ABCG1), although not significantly. In terms of genes related to fatty acid utilization, rhein did not change the expression of lipoprotein lipase (LPL) or $\beta 3$-adrenergic receptor ( $\beta 3$-AR) (Figure 3A). In skeletal muscle, rhein significantly decreased the expression of ACC1, and moderately decreased the expression of SREBP1c, FAS and SCD1, but hardly affected the expression of GLUT4 and IRS1. However, rhein increased the expression of peroxisome proliferator-activated receptor co-activator (PGC1 $\mathrm{a}$ ) and carnitine palmitoyltransferase-1a (CPT1a), genes associated with fatty acid $\beta$-oxidation, although the changes were not statistically significant (Figure 3B). In the liver, rhein significantly reduced the expression levels of SREBP1c, FAS, SCD1 and ACC2, but not GLUT2 or IRS2. Rhein moderately decreased the expression of the cholesterol clearance genes ABCA1, ABCG1 and CYP7A1, and significantly reduced the expression of the de novo cholesterol synthesis gene HMG CoA reductase (HMG-CoAR) (Figure 3C). In terms of markers of apoptosis, rhein significantly reduced the mRNA expression of caspase 3 in WAT, but not caspase 8 or caspase 9. Furthermore, rhein did not significantly affect the expression of caspase 3, 8 or 9 in muscle or liver (Supplementary Material: Figure S3). Taken together, these results indicate that rhein reprograms LXR target genes in WAT, muscle and liver.

\section{Rhein activates the gene expression of UCP I in BAT through LXR antagonism}

Adaptive thermogenesis in small mammals mostly takes place in BAT through the specific activity of UCP1 (7;8). Recently, Sheila Collins and her group reported that LXRa is a transcriptional repressor of UCP1 (21). Therefore, we investigated whether rhein enhances the expression of UCP1 to increase adaptive thermogenesis to counteract obesity. Interscapular BAT was obtained from wild-type (WT) mice fed with a high-fat diet or a high-fat diet together with rhein (by gavage), and was then subjected to quantitative RT-PCR. This analysis showed that rhein increased 
UCP1 mRNA levels by almost 3-fold, but did not affect the expression of $\beta 3$-AR (Figure 4A). To confirm the role of rhein in antagonism of LXR mediated repression of UCP1 expression, we analyzed UCP1 gene expression in BAT of LXR knockout $(\mathrm{KO})$ mice and found that rhein did not increase UCP1 expression in these mice (Figure 4B).

To further confirm that rhein could antagonize the repressive effect of LXR and thus increase UCP1 expression, we used HIB-1B immortalized brown adipocytes as a cell model. Real-time PCR showed that $25 \mu \mathrm{M}$ rhein increased the basal expression of UCP1. Notably, $1 \mu \mathrm{M}$ GW3965 decreased the level of UCP1 to $60 \%$ of the basal level, and this was reversed by rhein. This phenomenon was more pronounced when rhein was administered in combination with the adenylyl cyclase activator forskolin (FSK) (Figure 4C). Moreover, the electrophoretic mobility shift assay
(EMSA) showed that rhein reduced GW3965-induced binding of LXRa to the UCP1 promoter region (Figure $4 \mathrm{D})$, thus relieving transcriptional repression of LXR on UCP1, and allowing an increase in UCP1 expression.

Electron microscopic morphometric analysis showed that rhein markedly increased the number of mitochondria and decreased the size of lipid droplets in BAT (Figure 4E). These findings were confirmed by quantifying the number of mitochondria per unit nuclear DNA (Figure 4F). This phenomenon was consistent with the increased energy expenditure and the increased tolerance to cold following treatment with rhein (35). These data suggest that rhein activates UCP1 expression by counteracting LXR-mediated repression, and thus increases energy expenditure in BAT.
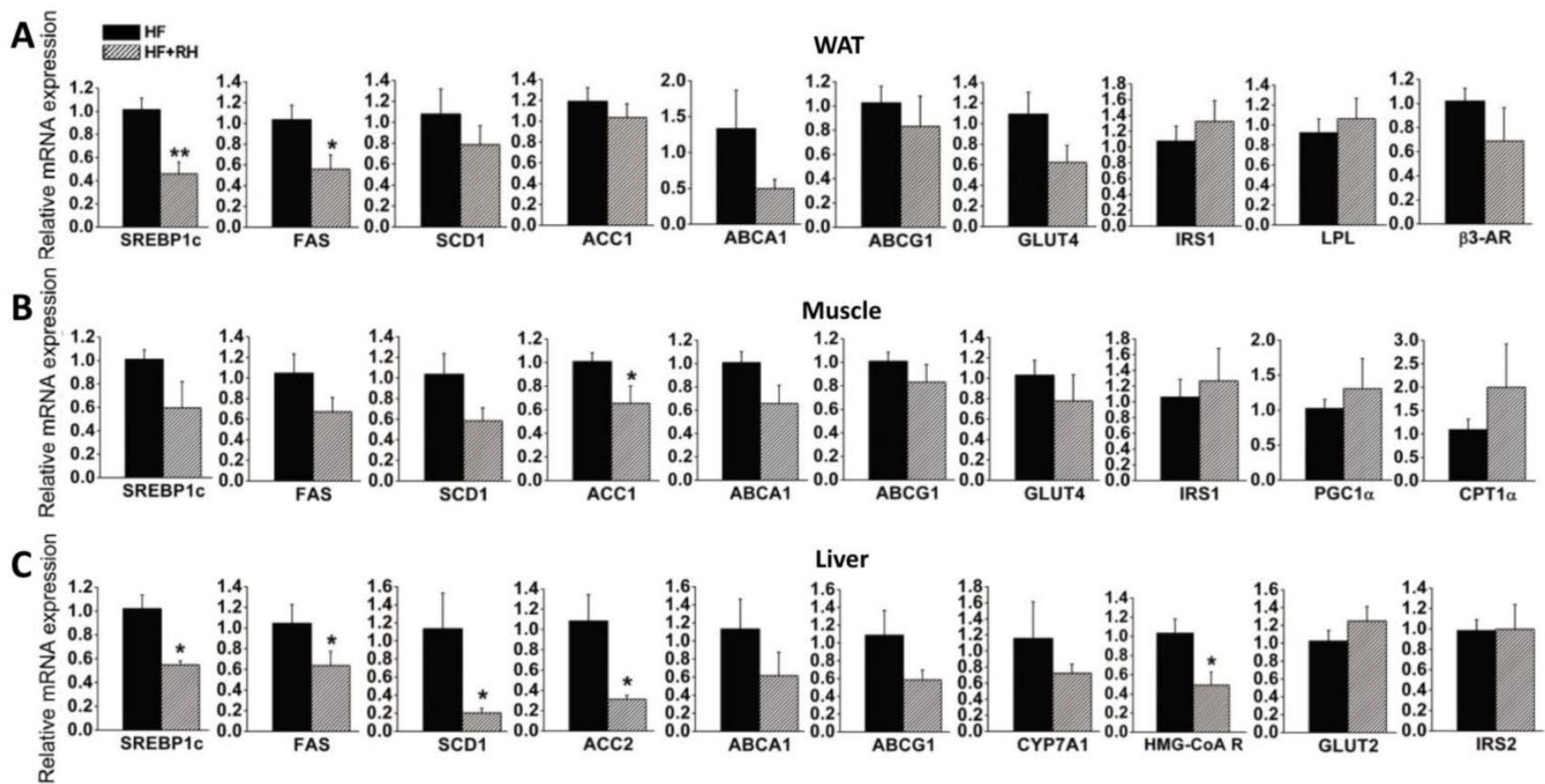

Figure 3 Rhein decreases the expression levels of liver $X$ receptor (LXR) target genes in white adipose tissue (WAT), muscle and liver in diet-induced obese mice. After administration of rhein for 4 weeks, total RNA was isolated from WAT, muscle and liver, and was subjected to quantitative real-time RT-PCR. Rhein decreased the expression of LXR target genes in WAT (A), muscle (B) and liver (C). The mRNA expression levels are shown as values in rhein-treated groups (striped bars, $H F+R H$ ) relative to the control (water) groups (black bars, $\mathrm{HF}$ ). Values are means \pm standard error of the mean for five mice per group. $* p<0.05, * * p<0.01$. 

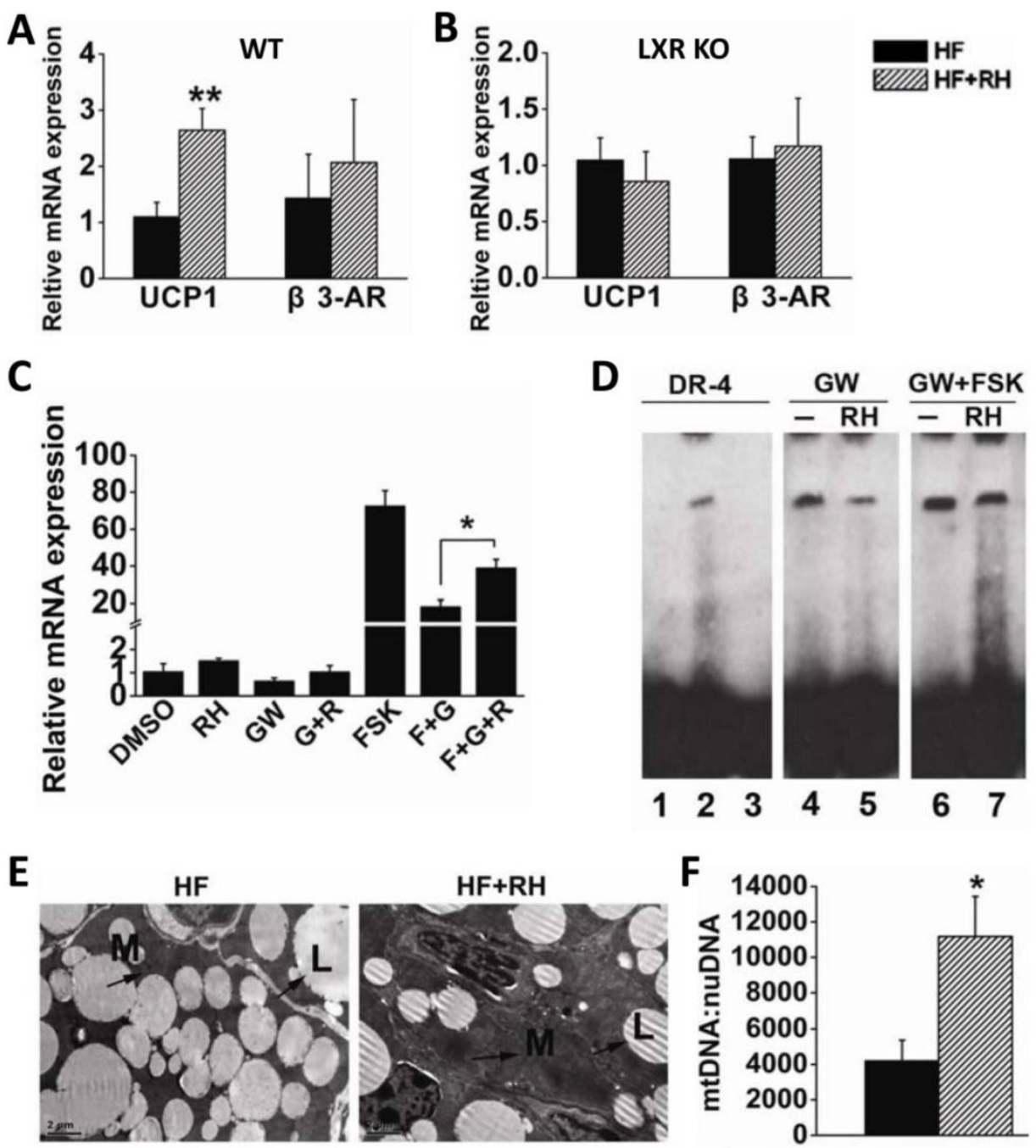

Figure 4 Rhein activates uncoupling protein I (UCPI) gene expression in brown adipose tissue (BAT) through LXR antagonism. (A) Rhein increased the expression of UCPI in BAT of high-fat diet-induced obese (DIO) wild-type mice. The mRNA expression levels are shown as values in rhein-treated groups (striped bars, $\mathrm{HF}+\mathrm{RH}$ ) relative to the control (water) groups (black bars, HF). Values are means \pm standard error of the mean (SEM) for five mice per group. $* * P<0.01$. (B) Rhein did not affect UCPI expression in BAT of high-fat DIO LXR knockout $(K O)$ mice. mRNA expression levels are shown as values in rhein-treated groups (striped bars, $\mathrm{HF}+\mathrm{RH}$ ) relative to the control (water) groups (black bars, HF). Values are means \pm SEM for five mice per group. (C) mRNA expression levels of UCPI in differentiated HIB-IB cells. HIB-IB cells were treated with forskolin (FSK, $10 \mu \mathrm{M})$, GW3965 (I $\mu \mathrm{M})$ or rhein $(25 \mu \mathrm{M})$ for $6 \mathrm{~h}$ and mRNA expression was determined by quantitative real-time PCR. Values are means \pm standard deviation of three independent experiments. (D) Differentiated HIB-IB cells were treated with the indicated compounds for $6 \mathrm{~h}$ and the nuclear extract (NE) was collected for electrophoretic mobility shift assays. Lane I: probe only; lane 2: probe plus NE [dimethyl sulfoxide (DMSO)]; lane 3: probe plus NE (DMSO) plus $100 \times$ competing probe; lane 4: probe plus NE (I $\mu M$ GW3965); lane 5: probe plus NE (I $\mu$ M GW3965 plus $25 \mu$ M rhein); lane 6: probe plus NE (I $\mu$ M GW3965 plus I0 $\mu$ M FSK); lane 7: probe plus NE (I $\mu$ M GW3965 plus $25 \mu$ M rhein plus $10 \mu$ M FSK). (E) Transmission electron microscopy images of BAT (magnification, 2550 $\times$ ) from high-fat DIO mice treated with water (HF) or rhein $(H F+R H)$. Arrows indicate the mitochondria $(M)$ and lipid droplets $(\mathrm{L})$. (F) Number of mitochondria per unit of nuclear DNA in BAT of high-fat DIO mice treated with rhein (striped bars) or water (black bars). Values are means \pm SEM for five mice per group. $* P<0.05$.

\section{Discussion}

Rhein is a small lipophilic molecule that is readily distributed following administration. Our previous study showed that rhein ameliorated fatty liver disease by promoting a negative energy balance, regulating hepatic lipogenesis, and exerting immunomodulatory effects in diet-induced obese (DIO) mice
(35). In the present study, we found that rhein is a mild but efficient and specific antagonist for LXRs based on four lines of evidence. First, the SPR assay for the interaction between rhein and LXR showed that rhein bound directly to both LXRa and LXR $\beta$. Second, rhein decreased the expression of LXR target genes stimulated by LXR agonist GW3965 in 3T3-L1 adipocytes and HepG2 cells in vitro. Third, rhein de- 
creased the expression of LXR target genes in WAT, muscle and liver in DIO mice. Fourth, rhein antagonized the repressive effects of LXRa on UCP1 expression and significantly increased UCP1 gene expression in BAT in DIO WT mice. However, rhein could not protect against high-fat diet-induced obesity in LXR KO mice.

Oral administration of rhein prevented fat accumulation in high-fat DIO mice (35). LXRs have been characterized as sterol sensors. Once activated, the body removes excess free cholesterol, increases the synthesis of fatty acids and triglyceride (TG), storing them in the liver and adipose tissue, and suppresses thermogenesis to favor the storage of lipid-rich energy. Once antagonized, the body tends to decrease the synthesis of fatty acids and TG, preventing their storage in liver and adipose tissue, and activates thermogenesis to favor energy expenditure to counteract obesity. Notably, these protective effects of rhein against obesity were not mediated by leptin signaling because rhein also decreased the body weight of $d b / d b$ mice, which lack the leptin receptor (Supplementary Material: Figure S4).

Obesity is a well-recognized risk factor for insulin resistance and type 2 diabetes. Although the expression of glucose metabolism-related genes such as GLUT-and IRS was not affected, rhein significantly improved glucose tolerance in DIO mice, which was confirmed in $d b / d b$ type 2 diabetes mice (35). Rhein decreased the expression of fatty acid synthesis genes in the liver, WAT and muscle, and had a systemic effect by increasing insulin sensitivity in these tissues. In particular, the increased expression of fatty acid oxidation-related genes such as CPT1a and PGC1a, combined with the absence of fatty acid synthesis in skeletal muscle, greatly reduced lipid accumulation. This could explain the improved glucose tolerance in rhein-treated mice because intramyocellular lipid accumulation is directly associated with insulin resistance $(39 ; 40)$.

We know that activation of LXRs can enhance cholesterol clearance in the liver and reverse cholesterol transport in the intestine by upregulating CYP7A1, ABCA1 and ABCG1 expression. Therefore, an LXR agonist could protect against atherosclerosis (41). The LXR antagonist rhein was reported to decrease serum total cholesterol (TC), high-density lipoprotein (HDL) and low-density lipoprotein (LDL) levels (35). Here, we showed that rhein decreased the expression levels of ABCA1 and ABCG1 in vitro and in vivo. Although rhein reduced the expression of cholesterol clearance genes, the hepatic expression of HMG-CoA R, which is associated with de novo cholesterol synthesis, was significantly downregulated.
This decrease might be responsible for the decrease in TC levels. This complicated phenomenon might be explained by the pleiotropic effects of rhein acting through targets other than LXRs. Although the LDL/HDL ratio was decreased by rhein (35), further studies are needed to investigate whether rhein has atherosclerotic activity.

We already know that thyroid hormone receptor (TR) and $\beta 3$-AR agonists can increase thermogenesis $(42 ; 43)$. However, rhein did not bind to the TRs in the SPR assay or activate the transcriptional activity of TRs in the luciferase reporter assay (Figure $1 G$ and $1 \mathrm{H}$, Supplementary Material: Figure S5). Although $\beta 3-\mathrm{AR}$ expression was elevated in BAT, we believe that the increase in thermogenesis and increased energy expenditure was not due to transactivation of $\beta 3-\mathrm{AR}$ by rhein for two reasons. First, rhein did not affect $\beta 3$-AR expression in WAT, a major target of $\beta 3-A R$ agonists. Second, chronic administration of a highly selective $\beta 3$-AR agonist, CL316,243, did not prevent high-fat diet-induced obesity or elevate the mRNA levels of $\beta 1-A R$ and $\beta 3-A R$ in BAT in C57BL/6J mice, as described by Collins et al. (44). Rhein might regulate UCP1 gene expression either directly or indirectly. Wang et al. reported that LXRa repressed the transcription of UCP1 and inhibited its expression (21). Our EMSA and real-time RT-PCR data showed that, as an antagonist of LXRs, rhein inhibited the binding of LXRa to the UCP1 promoter region and increased UCP1 expression. Accordingly, future studies should examine whether rhein directly increases UCP1 gene expression. Recently, Stulnig et al. reported a 2-fold decrease in UCP1 gene expression in BAT in WT mice, but no change in LXR KO mice treated with T0901317, an LXR agonist (45). Korach-Andre et al. showed that UCP1 mRNA and protein levels were highly induced in LXR $\alpha$ and LXR $\beta$ KO mice and repressed by GW3965 in BAT (46). Consistent with the above observations, our study found that rhein increased the expression of UCP1 by 3-4-fold in BAT in WT but not in LXR KO mice.

Paradoxically, antagonism of LXR may protect against high-fat diet-induced obesity but no such benefits are observed in LXR KO mice. Challenge with a high-fat diet increases ectopic UCP1 expression in muscle and WAT in LXR KO mice, and also causes aberrant production of thyroid hormone in the liver, thus increasing energy expenditure. However, fatty acid synthesis in muscle (3-fold) and WAT (4-fold) had increased to compensate for the weight gain caused by the high-fat diet (47). Thus, the phenotype of mice treated with an LXR antagonist does not fully mimic the phenotype of mice with knockout of both LXRa and LXR $\beta$, This suggests that the effects of 
acute and partial inhibition of LXRs differ from those of lifelong absence of LXRs and that there may be some compensatory mechanisms in this phenotype.

In conclusion, rhein is a natural compound that may have pleiotropic effects and multiple targets. Our study demonstrated the effects of rhein on obesity and related metabolic disorders through LXR antagonism and the unique effects of rhein on UCP1 expression in BAT.

\section{Materials and Methods}

\section{Reagents and plasmids}

Insulin, dexamethasone, IBMX, T3, rhein, chenodeoxycholic acid, 9-cis-retinoic acid, PCN, GW3965, GW0742, WY14643 and rosiglitazone were obtained from Sigma-Aldrich (St. Louis, MO). The pcDNA3 plasmids for the LBD of LXRa/ $\beta$, the GAL4-luc plasmids, the pcDNA3 plasmids for $\mathrm{LXR \alpha} / \beta$, the pTK-2 $\times$ LXRE-ABCA1-luc plasmids and the expression plasmids for RXR were kind gifts from Dr. Saez (The Scripps Research Institute, La Jolla, CA). The plasmids pET11b-hLXRa-LBD and pRSETA-hLXR $\beta$-LBD were kindly provided by Dr. Mangelsdorf (Southwestern Medical Center, Dallas, TX) and the PCMX plasmids for the LBDs of farnesoid $X$-activated receptor (FXR), pregnane $x$ receptor (PXR) and RXR were kindly provided by Dr. Moore (Baylor College of Medicine, Houston TX). The plasmids TRE-pal-luc, pcDNA3.1-TRa1 and pcDNA3.1-TR $\beta 1$ were kindly provided by Dr. Hao Ying (Institute for Nutritional Sciences, Shanghai, China).

\section{SPR assay}

The binding affinity of rhein towards LXRa-LBD, LXR $\beta$-LBD, TRa and TR $\beta$ was assayed using an SPR-based Biacore 3000 instrument (GE Healthcare, Uppsala, Sweden). The equilibrium dissociation constant $\left(\mathrm{K}_{\mathrm{D}}\right)$ was determined by analysis of the sensorgram curves obtained at six different concentrations using BIA evaluation software version 3.1 (Biacore) with a 1:1 Langmuir binding fitting model.

\section{Animals}

Female C57BL/6J mice (8-12 weeks old) were obtained from the SLAC Laboratory (Shanghai, China). Female $d b / d b$ (C57BL BKS cg-M ${ }^{+/+}$lepr $^{-/-}$) mice aged 8-10 weeks were from the Jackson Laboratory (Bar Harbor, ME). Female LXRa/ $\beta$ knockout (LXR ${ }^{-/-}$; C57BL/6J background) and WT mice were gifts from Dr. Mangelsdorf (Southwestern Medical Center). The mice were given a high-fat diet $(60 \%$ of calories derived from fat; D12492) or a control diet (10\% of calories derived from fat; D12450B) (Research Diets, New
Brunswick, NJ). All mice were maintained in a temperature-controlled facility $\left(23^{\circ} \mathrm{C}\right)$ with a $12-\mathrm{h}$ light/dark cycle and were given free access to food and water. Body weight and food intake were measured every 2 days and the treatments lasted for 4 weeks. Rhein $(150 \mathrm{mg} / \mathrm{kg})$ in water or water alone in the control group were administered by gavage every day at 2:00 pm. All animal protocols were approved by the Shanghai Institutes for Biological Sciences of the Chinese Academy of Sciences.

\section{In vivo analysis}

Body composition was measured by nuclear magnetic resonance imaging (Bruker Minispec mq 7.5, Karlsruhe, Germany). Oxygen consumption and physical activity were determined using CLAMS (Columbus Instruments, Columbus, $\mathrm{OH}$ ) according to the manufacturer's instructions. Rectal temperature of the mice was measured using a microprobe thermometer (Physitemp Instruments, Clifton, NJ) after placing the mice at $4^{\circ} \mathrm{C}$ for 4 hours.

\section{Histologic examinations and mtDNA quanti- fication}

Frozen WAT samples were embedded in OCT, sliced and stained with hematoxylin-eosin (H\&E). BAT sections were embedded and imaged using a Philips Tecbai-12 Biotwin Transmission Electron Microscopy (FEI Company, Eindhoven, The Netherlands). Total DNA was isolated from BAT using standard protocols. To quantify the amount of mtDNA present per nuclear genome, we used the following forward and reverse primers: mtDNA, 5'-CCTATCACCCTTGCCATCAT-3' and 5'-GAGGCT GTTGCTTGTGTGAC-3'; nuclear DNA, 5'-ATGGAA AGCCTGCCATCATG- $3^{\prime}$ and $5^{\prime}$-TCCTTGTTGTTCAG CATCAC-3'. To quantify the relative copy number differences, we determined the difference in threshold amplification between mtDNA and nuclear DNA using the $\Delta \Delta \mathrm{C}(\mathrm{t})$ method.

\section{Cell culture, transient transfection and lucif- erase reporter assays}

HepG2, 3T3-L1 and 293T cell lines obtained from the ATCC (Manassas, VA) were grown in Dulbecco's modified Eagle's medium containing 10\% fetal bovine serum (FBS) at $37^{\circ} \mathrm{C}$ with $5 \% \mathrm{CO}_{2}$. To induce differentiation, 2 days after confluence (designated day 0 ), 3T3-L1 cells were cultured in differentiation medium (DM) containing $10 \%$ FBS plus $10 \mu \mathrm{g} / \mathrm{ml}$ insulin, 0.5 $\mu \mathrm{M}$ dexamethasone and $0.5 \mathrm{mM}$ IBMX for 2 days, and then switched to post-DM containing $10 \% \mathrm{FBS}$ and 10 $\mu \mathrm{g} / \mathrm{ml}$ insulin. The medium was replaced every 2 days until day 7. Transfection of $293 \mathrm{~T}$ cells was per- 
formed using Lipofectamine 2000 (Invitrogen, Carlsbad, CA) with the indicated plasmids or control vectors. Luciferase reporter assays were carried out using the Dual-Luciferase Reporter Assay System (Promega, Madison, WI) and transfection efficiency was normalized by Renilla luciferase activity. Fully differentiated HIB-1B brown preadipocytes were treated for 6 h with DMSO, FSK $(10 \mu \mathrm{M})$, GW3965 $(1 \mu \mathrm{M})$, rhein $(25$ $\mu \mathrm{M})$, GW3965 plus rhein, FSK plus GW3965 and FSK plus GW3965 plus rhein for gene analysis and EMSA.

\section{Gene analysis}

Total RNA was extracted from cells or frozen tissues and then reverse transcribed into first-strand cDNA (Qiagen, Hilden, Germany). Gene expression levels were analyzed by quantitative RT-PCR using the ABI 7500 system (Applied Biosystems, Foster City, CA). The mRNA levels were normalized using $\beta$-actin as an internal control.

\section{EMSA}

Nuclear extracts were prepared using the NE-PER Nuclear and Cytoplasmic Extraction Kit (Pierce Biotechnology, Rockford, IL). Double-stranded gel shift probes were end-labeled with biotin. The binding reactions were analyzed using the LightShift Chemiluminescent EMSA Kit (Pierce Biotechnology). The oligonucleotide probe sequence was DR-4 5'-TCTACAGCGTCACAGAGGGTCAGTCAC$3^{\prime}$.

\section{Statistical analyses}

Values are expressed as means \pm SEM for experiments in mice. The two-tailed Student's t test was used to compare two groups, and one-way analysis of variance followed by the Student-Newman-Keuls test was used to compare three or more groups.

\section{Supplementary Material}

Fig.S1 - S5.

http://www.biolsci.org/v08p1375s1.pdf

\section{Acknowledgments}

We thank Dr. David J. Mangelsdorf (Southwestern Medical Center) for the LXRa/ $\beta$ knockout mice and for advice on the discussion. We also thank Dr. Sheila Collins (Duke University Medical Center) and Bruce M. Spiegelman (Harvard Medical School) for the HIB-1B cell line. The authors wish to acknowledge Dr. Nicholas Smith of Edanz Writing for editorial support and help in preparing the manuscript.

\section{Funding}

This work was supported in part by the Ministry of Science and Technology of China (2009CB919000), the Chinese Academy of Sciences (KSCX2-YW-G-057), and the Science and Technology Commission of Shanghai Municipality (07JC14011 and 07DJ14005).

\section{Competing Interests}

The authors have declared that no competing interest exists.

\section{References}

1. Ravussin E, Kozak LP. Have we entered the brown adipose tissue renaissance? Obes.Rev. 2009;10:265-8.

2. Spiegelman BM, Flier JS. Obesity and the regulation of energy balance. Cell 2001;104:531-43.

3. Kahn BB, Flier JS. Obesity and insulin resistance. I.Clin.Invest 2000;106:473-81.

4. Berge KE, von BK, Lutjohann $\mathrm{D}$ et al. Heritability of plasma noncholesterol sterols and relationship to DNA sequence polymorphism in ABCG5 and ABCG8. J.Lipid Res. 2002;43:486-94.

5. Hedley AA, Ogden CL, Johnson CL, Carroll MD, Curtin LR, Flegal KM. Prevalence of overweight and obesity among US children, adolescents, and adults, 1999-2002. JAMA 2004;291:2847-50.

6. Rosen ED, Spiegelman BM. Adipocytes as regulators of energy balance and glucose homeostasis. Nature 2006;444:847-53.

7. Lowell BB, Susulic V, Hamann A et al. Development of obesity in transgenic mice after genetic ablation of brown adipose tissue. Nature 1993;366:740-2.

8. Cannon B, Nedergaard J. Brown adipose tissue: function and physiological significance. Physiol Rev. 2004;84:277-359.

9. Nedergaard J, Bengtsson T, Cannon B. Unexpected evidence for active brown adipose tissue in adult humans. Am.J.Physiol Endocrinol.Metab 2007;293:E444-E452.

10. Virtanen KA, Lidell ME, Orava J et al. Functional brown adipose tissue in healthy adults. N.Engl.J.Med. 2009;360:1518-25.

11. van Marken Lichtenbelt WD, Vanhommerig JW, Smulders NM et al. Cold-activated brown adipose tissue in healthy men. N.Engl.J.Med. 2009;360:1500-8.

12. Cypess AM, Lehman S, Williams G et al. Identification and importance of brown adipose tissue in adult humans. N.Engl.J.Med. 2009;360:1509-17.

13. Apfel R, Benbrook D, Lernhardt E, Ortiz MA, Salbert G, Pfahl M. A novel orphan receptor specific for a subset of thyroid hormone-responsive elements and its interaction with the retinoid/thyroid hormone receptor subfamily. Mol.Cell Biol. 1994;14:7025-35.

14. Song C, Kokontis JM, Hiipakka RA, Liao S. Ubiquitous receptor: a receptor that modulates gene activation by retinoic acid and thyroid hormone receptors. Proc.Natl.Acad.Sci.U.S.A 1994;91:10809-13.

15. Willy PJ, Umesono K, Ong ES, Evans RM, Heyman RA, Mangelsdorf DJ. LXR, a nuclear receptor that defines a distinct retinoid response pathway. Genes Dev. 1995;9:1033-45.

16. Chawla A, Repa JJ, Evans RM, Mangelsdorf DJ. Nuclear receptors and lipid physiology: opening the X-files. Science 2001;294:1866-70.

17. Schultz JR, Tu H, Luk A et al. Role of LXRs in control of lipogenesis. Genes Dev. 2000;14:2831-8.

18. Peet DJ, Turley SD, Ma W et al. Cholesterol and bile acid metabolism are impaired in mice lacking the nuclear oxysterol receptor LXR alpha. Cell 1998;93:693-704.

19. Janowski BA, Willy PJ, Devi TR, Falck JR, Mangelsdorf DJ. An oxysterol signalling pathway mediated by the nuclear receptor LXR alpha. Nature 1996;383:728-31.

20. Mitro N, Mak PA, Vargas L et al. The nuclear receptor LXR is a glucose sensor. Nature 2007;445:219-23.

21. Wang $\mathrm{H}$, Zhang $\mathrm{Y}$, Yehuda-Shnaidman $\mathrm{E}$ et al. Liver $\mathrm{X}$ receptor alpha is a transcriptional repressor of the uncoupling protein 1 gene and the brown fat phenotype. Mol.Cell Biol. 2008;28:2187-200.

22. Malaguti C, Vilella CA, Vieira KP, Souza GH, Hyslop S, Zollner RL. Diacerhein downregulate proinflammatory cytokines expression and decrease the autoimmune diabetes frequency in nonobese diabetic (NOD) mice. Int.Immunopharmacol. 2008;8:782-91.

23. Jia $\mathrm{ZH}$, Liu $\mathrm{ZH}$, Zheng JM, Zeng $\mathrm{CH}$, Li LS. Combined therapy of rhein and benazepril on the treatment of diabetic nephropathy in $\mathrm{db} / \mathrm{db}$ mice. Exp.Clin.Endocrinol.Diabetes 2007;115:571-6. 
24. Choi SB, Ko BS, Park SK, Jang JS, Park S. Insulin sensitizing and alpha-glucoamylase inhibitory action of sennosides, rheins and rhaponticin in Rhei Rhizoma. Life Sci. 2006;78:934-42.

25. Hsia TC, Yang JS, Chen GW et al. The roles of endoplasmic reticulum stress and $\mathrm{Ca} 2+$ on rhein-induced apoptosis in A-549 human lung cancer cells. Anticancer Res. 2009;29:309-18.

26. Heo SK, Yun HJ, Park WH, Park SD. Rhein inhibits TNF-alpha-induced human aortic smooth muscle cell proliferation via mitochondrial-dependent apoptosis. J.Vasc.Res. 2009;46:375-86.

27. Shi $P$, Huang $Z$, Chen $G$. Rhein induces apoptosis and cell cycle arrest in human hepatocellular carcinoma BEL-7402 cells. Am.J.Chin Med. 2008;36:805-13.

28. Kuo PL, Hsu YL, Ng LT, Lin CC. Rhein inhibits the growth and induces the apoptosis of Hep G2 cells. Planta Med. 2004;70:12-6.

29. Kagedal K, Bironaite D, Ollinger K. Anthraquinone cytotoxicity and apoptosis in primary cultures of rat hepatocytes. Free Radic.Res. 1999;31:419-28.

30. Wang C, Jiang Z, Yao J et al. Participation of cathepsin B in emodin-induced apoptosis in HK-2 Cells. Toxicol.Lett. 2008;181:196-204.

31. Chantre P, Cappelaere A, Leblan D, Guedon D, Vandermander J, Fournie B. Efficacy and tolerance of Harpagophytum procumbens versus diacerhein in treatment of osteoarthritis. Phytomedicine. 2000;7:177-83.

32. Leblan D, Chantre P, Fournie B. Harpagophytum procumbens in the treatment of knee and hip osteoarthritis. Four-month results of a prospective, multicenter, double-blind trial versus diacerhein. Joint Bone Spine 2000;67:462-7.

33. Nguyen M, Dougados M, Berdah L, Amor B. Diacerhein in the treatment of osteoarthritis of the hip. Arthritis Rheum. 1994;37:529-36.

34. Taccoen A, Berdah L. [Diacetylrhein, a new therapeutic approach of osteoarthritis]. Rev.Rhum.Ed Fr. 1993;60:83S-6S.

35. Sheng X, Wang M, Lu M, Xi B, Sheng H, Zang YQ. Rhein ameliorates fatty liver disease through negative energy balance, hepatic lipogenic regulation, and immunomodulation in diet-induced obese mice. Am.J.Physiol Endocrinol.Metab 2011;300:E886-E893.

36. Ueda $Y$, Yamagishi $\mathrm{T}$, Samata $\mathrm{K}$ et al. A novel low molecular weight antagonist of vascular endothelial growth factor receptor binding: VGA1155. Mol.Cancer Ther. 2003;2:1105-11.

37. Vassilev LT, Vu BT, Graves B et al. In vivo activation of the $\mathrm{p} 53$ pathway by small-molecule antagonists of MDM2. Science 2004;303:844-8.

38. Asano K, Ono A, Hashimoto S, Inoue T, Kanno J. Screening of endocrine disrupting chemicals using a surface plasmon resonance sensor. Anal.Sci. 2004;20:611-6.

39. Oakes ND, Bell KS, Furler SM et al. Diet-induced muscle insulin resistance in rats is ameliorated by acute dietary lipid withdrawal or a single bout of exercise: parallel relationship between insulin stimulation of glucose uptake and suppression of long-chain fatty acyl-CoA. Diabetes 1997;46:2022-8.

40. Shimabukuro M, Koyama K, Chen G et al. Direct antidiabetic effect of leptin through triglyceride depletion of tissues. Proc.Natl.Acad.Sci.U.S.A 1997;94:4637-41.

41. Kalaany NY, Mangelsdorf DJ. LXRS and FXR: the yin and yang of cholesterol and fat metabolism. Annu.Rev.Physiol 2006;68:159-91.

42. $\mathrm{Hu}$ B, Jennings LL. Orally bioavailable beta 3-adrenergic receptor agonists as potential therapeutic agents for obesity and type-II diabetes. Prog.Med.Chem. 2003;41:167-94.

43. Grover GJ, Mellstrom K, Ye L et al. Selective thyroid hormone receptor-beta activation: a strategy for reduction of weight, cholesterol, and lipoprotein (a) with reduced cardiovascular liability. Proc.Natl.Acad.Sci.U.S.A 2003;100:10067-72.

44. Collins S, Daniel KW, Petro AE, Surwit RS. Strain-specific response to beta 3-adrenergic receptor agonist treatment of diet-induced obesity in mice. Endocrinology 1997;138:405-13.

45. Stulnig TM, Steffensen KR, Gao $\mathrm{H}$ et al. Novel roles of liver $\mathrm{X}$ receptors exposed by gene expression profiling in liver and adipose tissue. Mol.Pharmacol. 2002;62:1299-305.

46. Korach-Andre M, Archer A, Barros RP, Parini P, Gustafsson JA. Both liver-X receptor (LXR) isoforms control energy expenditure by regulating brown adipose tissue activity. Proc.Natl.Acad.Sci.U.S.A 2011;108:403-8.

47. Kalaany NY, Gauthier KC, Zavacki AM et al. LXRs regulate the balance between fat storage and oxidation. Cell Metab 2005;1:231-44. 